\title{
The Three-dimensional Structure of an lonotropic Glutamate Receptor Reveals a Dimer-of-dimers Assembly
}

\section{Willem Tichelaar ${ }^{1}$, Markus Safferling ${ }^{1}$, Kari Keinänen ${ }^{2}$, Holger Stark ${ }^{3}$ and Dean R. Madden ${ }^{1,4 *}$}

${ }^{1}$ Ion Channel Structure Group Max Planck Institute for Medical Research, Jahnstr. 29 D-69120 Heidelberg, Germany

${ }^{2}$ Department of Biological and Environmental Sciences, P.O. Box 56, FI-00014 University of Helsinki, Helsinki, Finland

${ }^{3}$ Max Planck Institute for Biophysical Chemistry, Am Fassberg 11, D-37077 Goettingen, Germany

${ }^{4}$ Department of Biochemistry Dartmouth Medical School 7200 Vail Building, Hanover NH 03755 USA

${ }^{*}$ Corresponding author

\begin{abstract}
The ionotropic glutamate receptors (iGluRs) represent a major family of ion channels whose quaternary structure has not yet been defined. Here, we present the three-dimensional structure of a fully assembled iGluR, determined at $\sim 20 \AA$ resolution by electron microscopy. Analysis of negatively stained single-particle images reveals the presence of 2-fold, but not 4 -fold, symmetry for these tetrameric channels, providing the first direct structural evidence for a dimer-of-dimers assembly. The receptor appears elongated, measuring $\sim 170 \AA \times 140 \AA \times 110 \AA$, with the 2-fold symmetry centered on its longitudinal axis. The overall molecular shape and symmetry suggest an orientation relative to the membrane and permit the identification of a putative transmembrane domain. Internal cavities located along the longitudinal axis may represent components of the ion conduction pathway.
\end{abstract}

(c) 2004 Elsevier Ltd. All rights reserved.

Keywords: ionotropic glutamate receptor; AMPA receptor; GluRB; ion channel gating; electron microscopy

\section{Introduction}

Glutamate is the major excitatory neurotransmitter in the central nervous system. Its cognate receptors include ligand-gated ion channels that play important roles in neuronal development, in synaptic plasticity, and in a variety of neurological disorders. Binding of agonist to an ionotropic glutamate receptor (iGluR) triggers the opening of an ion pore, allowing the flow of selected cations across the membrane. In the continuing presence of agonist, the receptor typically desensitizes, closing the channel. A subfamily of iGluRs exhibits high

Present address: M. Safferling, Bayer CropScience Aktiengesellschaft, R-TR, Building 6240, D-40789 Monheim, Germany.

Abbreviations used: iGluRs, ionotropic glutamate receptors; NTD, N-terminal domain; LBD, ligand-binding domain; ER, endoplasmic reticulum; MRA, multireference alignment; MSA, multivariate statistical analysis.

E-mail address of the corresponding author: drm0001@dartmouth.edu affinity for the synthetic agonist $\alpha$-amino-3hydroxy-5-methyl-4-isoxazolepropionic acid (AMPA), and is responsible for most fast excitatory synaptic signaling in the brain. However, despite their physiological importance, the subunit organization and symmetry of the iGluRs has remained elusive.

Like other iGluRs, AMPA receptors are assembled as homo- or heterotetramers of subunits. Referred to as GluRA-GluRD (or GluR1-GluR4), these subunits are themselves modular. ${ }^{2}$ They consist of two extracellular domains, each with homology to the bilobate bacterial periplasmic binding proteins. ${ }^{3}$ A membrane-distal N-terminal domain (NTD) is coupled to a membrane-proximal ligand-binding domain (LBD) that contains the agonist-binding site. The crystallographic structure of the LBD has been determined, revealing a bilobate structure that undergoes an agonistinduced cleft closure, the magnitude of which is correlated with the extent of channel activation. ${ }^{4}$ The LBD is connected in turn to the transmembrane domains, which exhibit partial homology to the 
primitive potassium channels. ${ }^{5}$ The $\mathrm{C}$ terminus is intracellular and mediates protein-protein interactions important in iGluR trafficking and localization. ${ }^{\circ}$

The following findings suggest that the component domains associate via dimeric interactions and that the receptors may not assemble as 4-fold symmetric tetramers. First of all, the LBD dimerizes weakly across an interface that appears to regulate channel desensitization. ${ }^{7,8}$ The LBD dimerization interaction has been visualized crystallographically: the domains associate via their membranedistal lobes in a "back-to-back" fashion, with their membrane-attachment sites pointing in the same direction and their binding clefts facing away from each other. ${ }^{4}$ In addition, Ayalon \& Stern-Bach ${ }^{9}$ used subunit chimeras to develop a model of sequential dimerization for receptor assembly, in which NTD compatibility is required for an initial obligate subunit pairing, while the LBDs and transmembrane domains stabilize a subsequent dimer-dimer association. This is consistent with the observation that the NTD dimerizes more strongly in solution than the LBD, ${ }^{10}$ although GluRD subunits lacking the NTD can still assemble to form functional channels. ${ }^{11}$ Further indirect support for a 2-fold symmetrical assembly is provided by the characteristics of channels formed by tandem subunits ${ }^{12}$ and by scanning cysteine mutagenesis data. ${ }^{13}$ The latter data also suggest that the 2-fold symmetry of the iGluRs may apply not only to the soluble extracellular domains, but also to at least that part of the transmembrane domains located in the extracellular leaflet of the plasma membrane, despite sequence similarity to the 4-fold symmetrical $\mathrm{K}^{+}$-channel family. ${ }^{5,14}$

Based on these biochemical and structural results, a working model for the assembly, activation and desensitization of the iGluRs has emerged. ${ }^{2}$ Nevertheless, to date, evidence for the shape and symmetry of fully assembled glutamate receptor ion channels remains indirect. Based on our ability to purify tetrameric GluRB homomers, ${ }^{15}$ we present here the first three-dimensional (3D) structure of an AMPA receptor, obtained by electron microscopy of negatively stained specimens and single-particle image analysis. The molecular envelope provides direct structural evidence for the overall 2-fold symmetry of the channel and suggests a probable orientation relative to the lipid bilayer.

\section{Results}

An electron micrograph of negatively stained GluRB is shown in Figure 1. Initial image analysis, $3 \mathrm{D}$ reconstruction and iterative refinement were carried out without imposing symmetry constraints (C1 symmetry). Examples of aligned singlemolecular images are displayed in Figure 2(A), and the final $C 1$ class averages to which each was assigned are shown in Figure 2(B). The first two

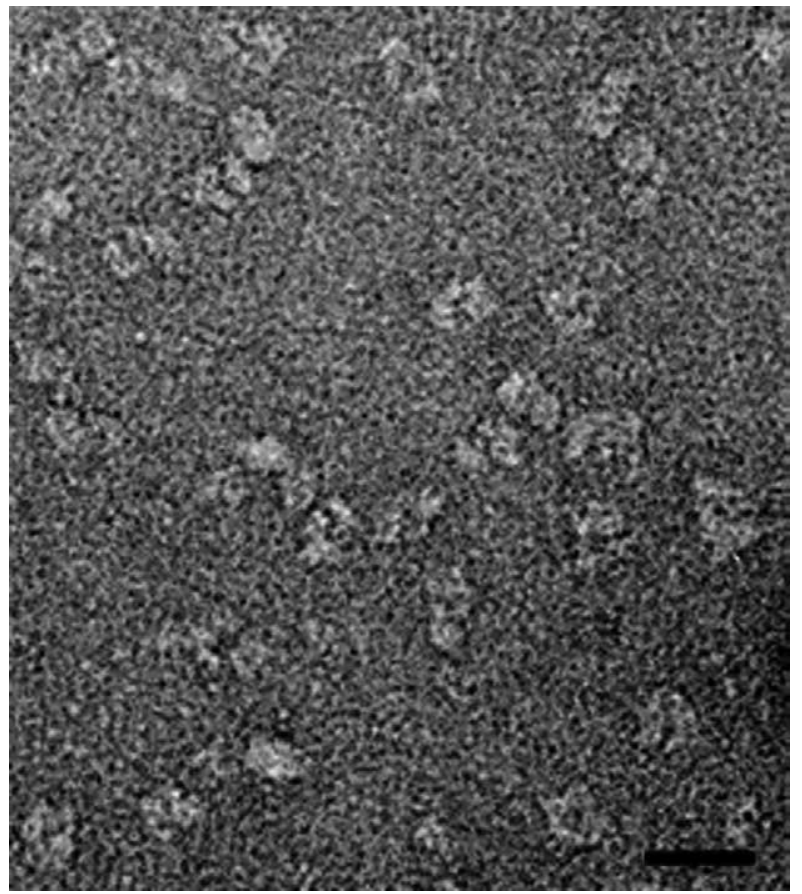

Figure 1. Overview of negatively stained GluRB. The scale bar represents $200 \AA$.

views in Figure 2(A) and (B) are the most frequently occurring ones, while the last two are relatively rare. As illustrated in the first three views, many projections are elongated and appear to be composed of three parts. One part, seen at the bottom of the projections as oriented in Figure 2, resembles a dumbbell (first image) or a ring surrounding a small stain accumulation (second and third images). At the other end of the particle a more compact, round feature is observed, which is smaller than the bottom part. Finally, between these two features, two arms are often seen that connect the domains at either end and encompass a relatively large central stain accumulation.

During initial image analysis (Figure 2(A)) and following convergence of the $C 1$ structure refinement (Figure 2(B)), mirror symmetrical views were observed frequently (e.g. third images), and approximately 2 -fold symmetrical views were observed occasionally (e.g. fifth images). In addition to the class averages, both eigenimage analysis and the $3 \mathrm{D}$ reconstruction itself also suggested 2-fold symmetry of the molecule (not shown). Therefore, a second round of refinement was initiated, imposing C2 symmetry constraints during Euler-angle determination and 3D reconstruction, until the $3 \mathrm{D}$ structure was stable and no further improvements in resolution could be attained.

Neither the class averages nor the eigenimages indicated the presence of 4-fold symmetry. However, due to the tetrameric nature of the receptor and partial sequence homology to the 4-fold symmetrical potassium channels, ${ }^{5}$ the applicability 


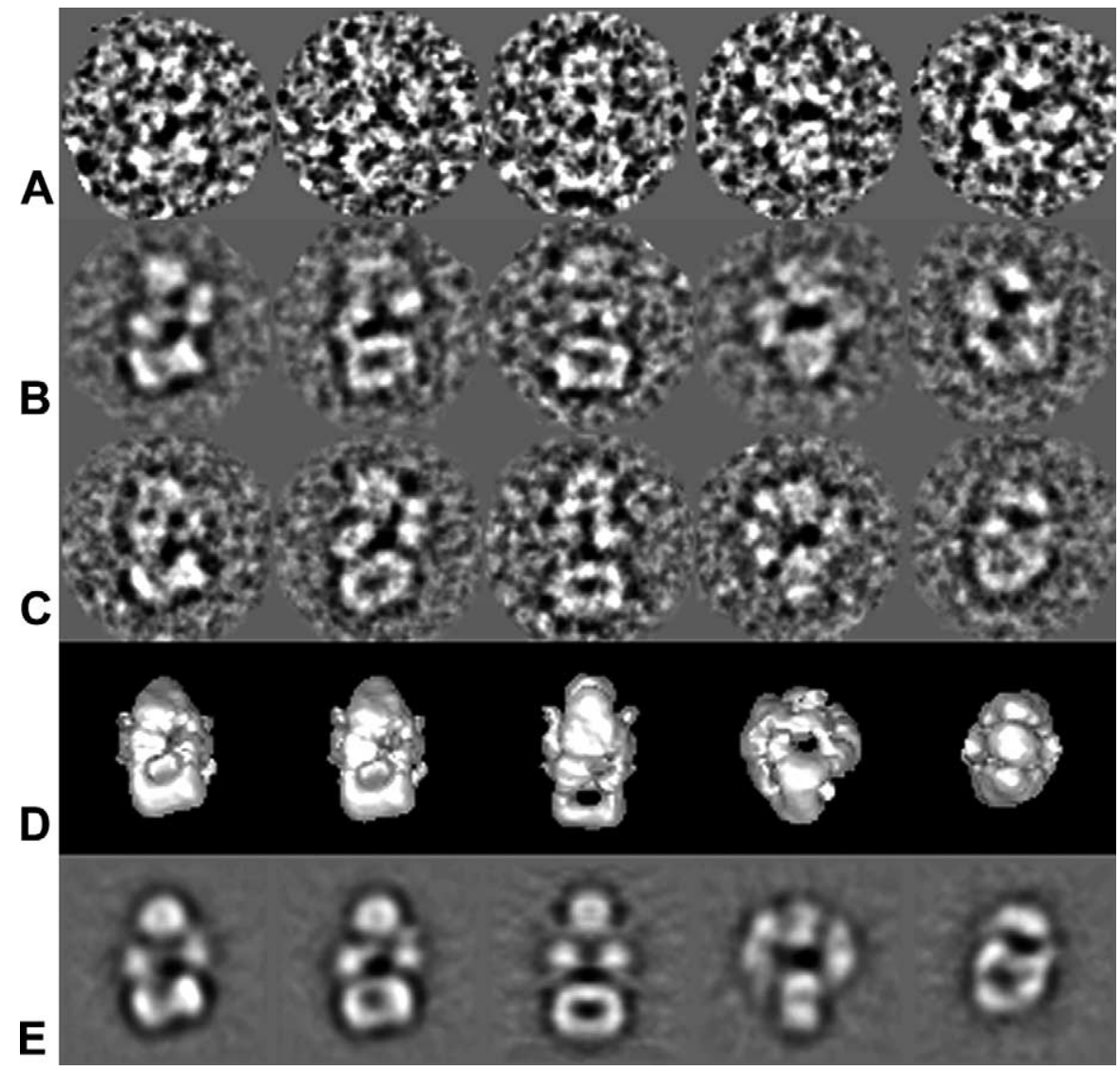

Figure 2. Different stages of the single-particle image analysis: (A) aligned single particles, (B) corresponding class averages at the end of the refinement under $C 1$ symmetry constraints, $(C)$ final class averages obtained under $C 2$ constraints, (D) surface representations of the final model in corresponding orientations, and (E) reprojections of the model. The views in the first two columns occur most frequently; those in the last two columns, relatively rarely. The edge of one frame (64 pixels) corresponds to $228 \AA$.

of $C 4$ constraints was tested at the final stage of the analysis. Using the class averages obtained in the final round of $\mathrm{C} 2$ refinement, additional 3D reconstructions were calculated assuming either $C 1$ or $C 4$ symmetry, and two assessments were carried out. The first one is depicted in Figure 3: reprojections of the unsymmetrized (C1) and the C2- and C4symmetrized models were compared with one another and with the corresponding input projections. Projections of the models obtained under $C 1$ and C2 symmetry agree well with each other and with the input projections. In contrast, the reprojections obtained under C4 symmetry differ markedly both from the input projections and from the reprojections in the case of $C 1$ and $C 2$, and also appear less well-defined (Figure 3). The average correlation coefficients between reprojections and input projections are $0.82,0.80$ and 0.68 with $C 1, C 2$ and $\mathrm{C} 4$ symmetries, respectively. Moreover, iterative refinements under $C 4$ symmetry constraints did not converge upon well-defined class averages and $3 \mathrm{D}$ reconstructions.

In a second assessment, the original dataset was aligned using reprojections of both the C2- and C4symmetrized models as references. The singlemolecular images were sorted according to the model to which they best correlated and then subjected to classification and $3 \mathrm{D}$ reconstruction under $C 2$ or $C 4$ symmetry constraints, respectively. This competitive alignment process was then repeated. Approximately one-third of the images aligned to C4-derived reprojections in the first cycle, most likely due to similarities between some reprojections at this stage, but this fraction dropped below one-fifth in subsequent rounds. The $C 2$ model remained constant during this test. On the basis of these results, we ruled out the possibility of 4-fold symmetry.

Class averages derived from the $\sim 80 \%$ of images that aligned with $C 2$ reprojections in the final round of competitive alignment are shown in Figure 2(C). They appear very similar to class averages obtained before symmetry constraints were imposed (Figure 2(B)). Using C2 symmetry constraints, relative projection angles were determined for a total of 350 class averages, and a final 3D reconstruction was calculated. Surface views of the resulting model are shown in Figure 2(D), in orientations corresponding to the views in Figure $2(\mathrm{~A})-(\mathrm{C})$. Reprojections are shown in Figure 2(E) and illustrate the agreement between the 3D map and the input projections.

Our model is presented in two stereo pairs (Figure $4(\mathrm{~A})$ ) and in two orthogonal views, a side 


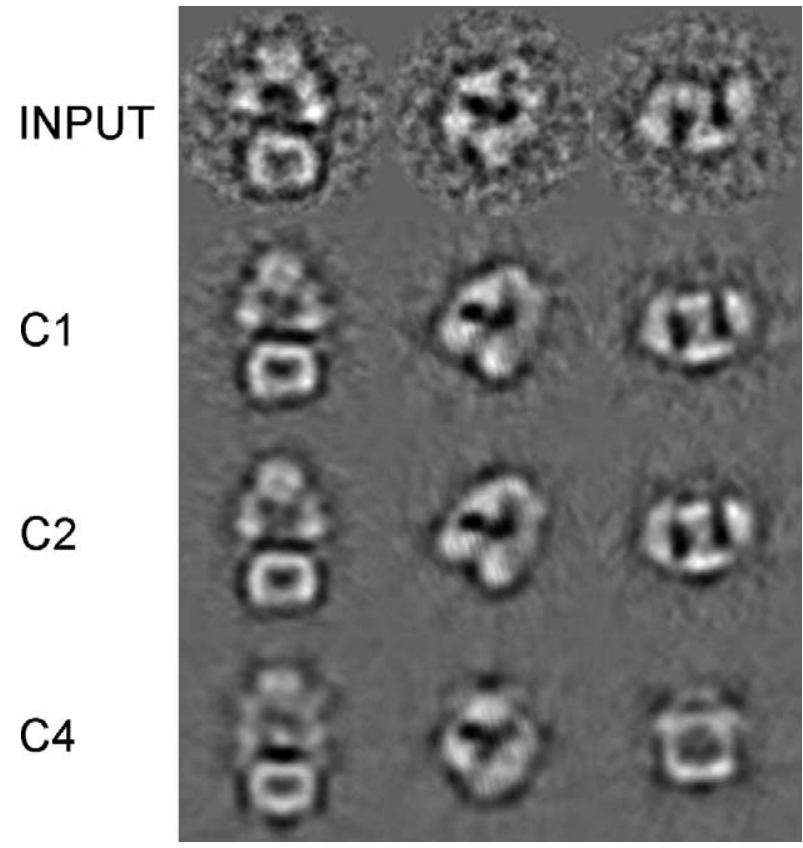

Figure 3. Symmetry assessment: class averages obtained once the image analysis under C2 symmetry constraints had converged, and used as input projections for $3 \mathrm{D}$ reconstructions ("Input") are shown together with corresponding reprojections computed from models that were reconstructed while imposing no symmetry (" $\mathrm{C} 1$ "), 2-fold ("C2"), or 4-fold ("C4") symmetry constraints, respectively. The edge of one frame (64 pixels) corresponds to $228 \AA$.

and a front view (Figure 4(B)). Roughly speaking, the model depicts GluRB as a hollow, approximately oblate rotational ellipsoid with 2-fold symmetry along its longitudinal axis and overall dimensions of $\sim 170 \AA \times 140 \AA \times 110 \AA$. The molecular envelope shown is contoured to yield a molecular mass of $\sim 540 \mathrm{kDa}$. Contouring the envelope to a volume corresponding to a GluRB dimer plus TX100 micelle $(\sim 285 \mathrm{kDa})$ yielded a disconnected molecular envelope (not shown), confirming the tetrameric nature of the samples used in the analysis. The particle consists of a $\sim 65 \AA$ high bottom part with a transverse hole and a $\sim 50 \AA$ high top part. These parts are separated by a central vestibule, as seen in cut-away views (Figure $4(\mathrm{C})$ ). Four arms run between the bottom and top parts. They are arranged around the central vestibule with a helical twist, and two of the arms are thicker and longer $(\sim 125 \AA)$ than the other two $(\sim 100 \AA$ ) (Figure $4(\mathrm{~B}))$. As shown in Figure 4 each short arm merges with one long arm before connecting with the top; a second lateral connection links each short arm to the other long arm.

The final Euler-angle distribution is shown in Figure 5. As determined by Fourier shell correlation, the resolution for the model is $\sim 17 \AA$

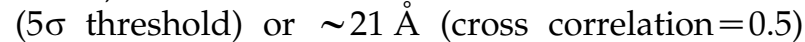
(Figure 6).

\section{Discussion}

The electron microscopic model shown in Figure 4 represents the first 3D structure of a fully assembled tetrameric glutamate receptor ion channel. The associated single-particle image analysis also constitutes the first direct structural evidence for the overall 2-fold symmetry of the iGluRs, supporting a dimer-of-dimers molecular architecture, consistent with indirect evidence based on the association properties of their constituent domains ${ }^{4,9,10}$ and on the electrophysiological properties of tandem and mutant iGluR subunits. ${ }^{12,13}$ The dimer-of-dimers symmetry supports proposed working models of iGluR function, in which agonist-induced cleft closure in the LBD forces the transmembrane domains apart across component dimer interfaces. ${ }^{2,4}$

Several considerations suggest that the transmembrane domains are located at the bottom of the molecular envelope as shown in Figure 4(B). First, the molecule is most likely oriented in the membrane with its longitudinal, 2-fold symmetry axis perpendicular to the plane of the membrane. Second, the subunit mass distribution $(\sim 80 \mathrm{kDa}$ extracellular; $\sim 10 \mathrm{kDa}$ intracellular) indicates that the transmembrane domains must be located at one end of the structure along the 2-fold axis. The bottom part of the structure, shown superimposed on a lipid bilayer in Figure 4(B), is distinct from the middle and top parts, is sufficiently thick to span a lipid bilayer, and has a relatively small crosssection, in accordance with the small transmembrane "footprint" of the intact channel (for which in total only twelve transmembrane domains are predicted). The other end of the molecular structure fulfills none of these criteria. The bottom part would also include the short cytoplasmic C-terminal domains of the four subunits.

If this assignment is correct, the remainder of the particle would contain the monomers' extracellular domains, with the LBDs arranged around a central "vestibule", and the NTDs clustered above them. Based on molecular volume considerations, the axial density located at the top of the particle could accommodate one but not both lobes of the presumably bilobate NTDs. Thus, the NTDs would extend down into the arms that connect the top and bottom parts of the molecular envelope, which would also contain the LBDs. Attempts to fit the known crystallographic structure of the LBD into the molecular envelope did not lead to a unique docking solution, most likely because the domain represents only $\sim 7 \%$ of the molar mass of the receptor. In analogy to the structure of the acetylcholine receptor, ${ }^{16}$ the molecular cavities located along the 2-fold symmetry axis and associated openings to the side (Figure $4(\mathrm{C})$ ) may represent components of the ion conduction pathway.

The molecular envelope tapers significantly towards the postulated transmembrane end (Figure 4(B), top). This tapering implies that the 2-fold symmetry axis of each component subunit dimer is 


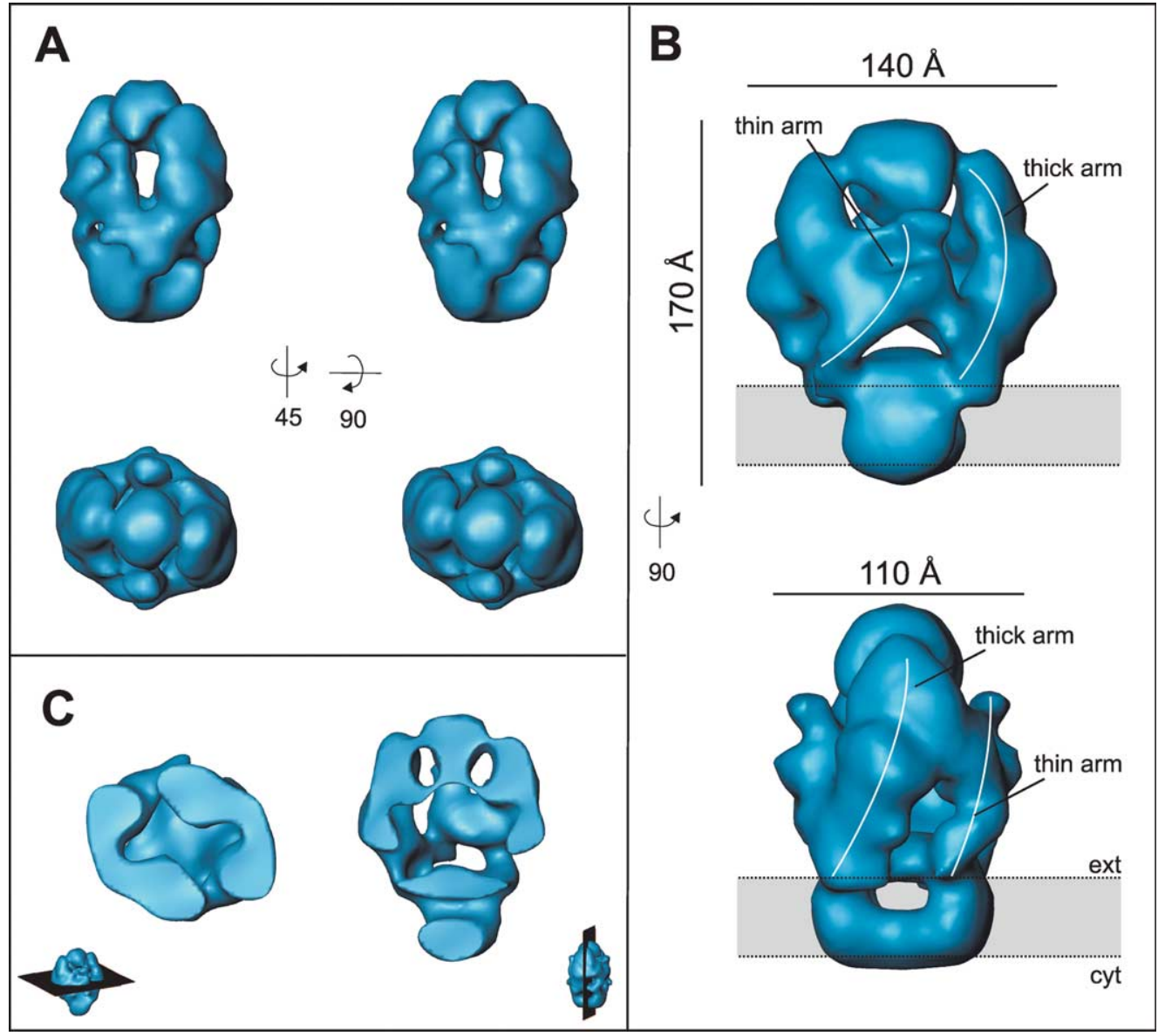

Figure 4. Representations of the 3D structure of the GluRB ionotropic glutamate receptor: (A) stereo views, (B) orthogonal side and front views, (C) cut-away views. The lower stereo pair in (A) represents a top view of the molecule as it would appear looking down on the membrane from the synaptic cleft. The upper stereo pair in (A) shows a side view of the molecule, which is offset $45^{\circ}$ around a vertical axis relative to the views shown in (B). In (B), the proposed location of the membrane is indicated by a shaded rectangle at the bottom of each figure, perpendicular to the receptor's longitudinal, 2-fold symmetry axis and perpendicular to the image plane. ext=extracellular; cyt=cytosolic; the thickness of the membrane as drawn is $36 \mathrm{~A}$. In (C), horizontal and vertical cut-away views of the central vestibule are shown, together with inset representations of the corresponding cutting planes.

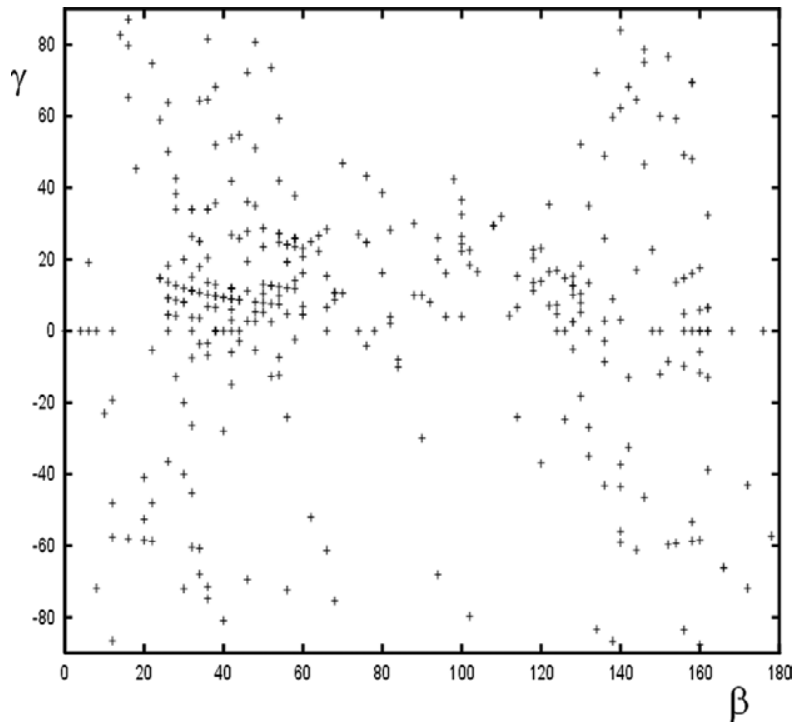

Figure 5. Euler-angle distribution of the 350 class averages incorporated in the model in Figure 4. not parallel with the overall 2-fold symmetry axis relating them in the tetrameric receptor (vertical in Figure 4(B)). As a result, addition of further subunit dimers to the outside of the assembled receptors could be suppressed, explaining why the secondary dimerization does not propagate indefinitely to form extended ribbons of dimers. Based on symmetry considerations, there are also two distinct subunit-packing environments within the tetramer, reflected by the occurrence of thin and thick arms in our model, for example. When studied at higher resolution, this non-equivalence may prove to have functional implications for the mechanisms of channel gating and desensitization.

Although there have been reports that GluRB homomers can be retained in the endoplasmic reticulum (ER) as dimers in human tissue, ${ }^{17,18}$ hydrodynamic studies ${ }^{15}$ and contouring experiments with the EM model confirm a tetrameric assembly of the insect-cell expressed homomers described here. Moreover, similar hydrodynamic behavior $^{10}$ and EM projection images (not shown) 


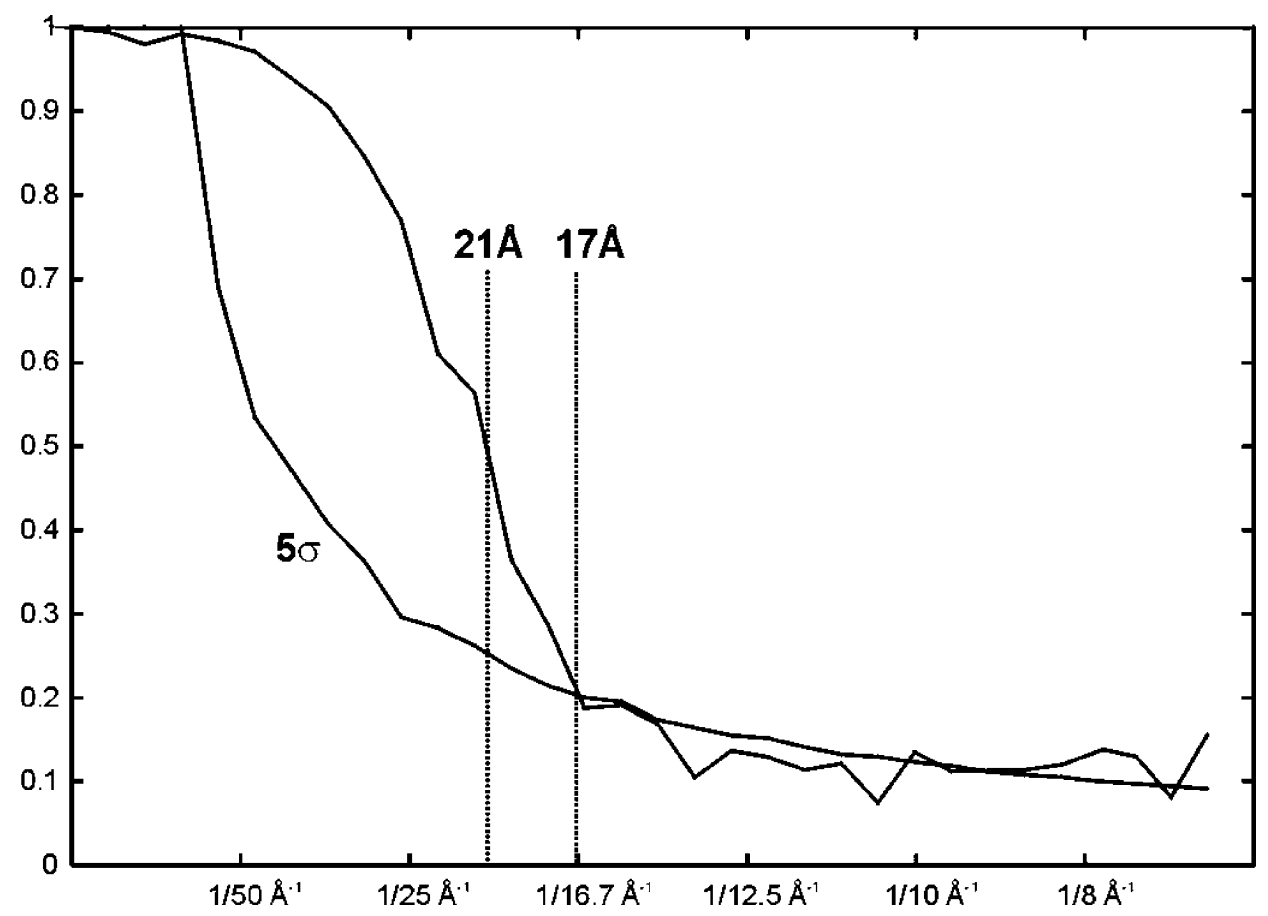

Figure 6. Fourier shell correlation between two models, obtained after splitting the final set of class averages in two halves. The FSC crosses the $5 \sigma$ significance curve, corresponding to the expected noise level, at $17 \AA$. The correlation is equal to 0.5 at $21 \AA$.

are observed for GluRD homomers, which do not share the pore-loop Arg implicated in ER retention. $.7,18$

From the homology of the iGluR transmembrane domains to the 4 -fold symmetric bacterial $\mathrm{K}^{+}$channels it has been concluded that there is a symmetry transition in the iGluR between the (2-fold symmetric) extracellular domains and the (putatively 4-fold symmetric) transmembrane domain. ${ }^{2,7}$ However, iGluR gating and ion selectivity are different from those of the potassium channels: iGluRs have a relatively large pore that does not select strongly among monovalent cations, they lack the inner-helix hinge glycine conserved in all potassium channels, ${ }^{19}$ and their gating is not affected by mutagenesis of other inner helix glycine residues. ${ }^{20}$ Finally, the effects of cadmium on hybrid iGluR composed of wild-type and Cys-mutant subunits at different ratios are best matched by model calculations assuming a pairwise association of subunits, which would be consistent with 2-fold symmetry extending at least into the upper third of the channel domain. ${ }^{13}$ In our structure, the 2-fold symmetry appears to apply throughout the molecular envelope, including the entire transmembrane region. Here, however, the envelope also reflects the contribution of the detergent micelle, whose shape may be influenced by the arrangement of the extracellular domains and could thus mask the symmetry of the protein's transmembrane domains.

In addition to providing first insights into the overall shape, symmetry and dimensions of an intact AMPA receptor, our model provides an anchor for more detailed EM analysis of iGluR structure/function relationships, including domain localization by antibody mapping ${ }^{21,22}$ and the application of higher resolution specimen preparation and imaging techniques. The channel has been imaged in the absence of agonist and is competent to bind ligand, so the structure presented most likely corresponds to the resting state. It is conceivable that the arrangement of the iGluR pore may change depending on the functional state of the channel; in the acetylcholine receptor, the pseudo-5-fold symmetry of the ligand-binding domains is broken in the ligand-free resting state, and restored upon channel activation. ${ }^{23}$ It will therefore be important to study the iGluR in defined, conformationally homogeneous functional states in order to understand the mechanisms of channel activation and desensitization.

\section{Materials and Methods}

GluRB tetramers were expressed and isolated to homogeneity as described. ${ }^{15}$ Samples in $25 \mathrm{mM}$ Tris ( $\mathrm{pH}$ 7.4), $636 \mathrm{mM} \mathrm{NaCl}, 2.7 \mathrm{mM} \mathrm{KCl}, 10 \%$ (w/v) glycerol, $0.1 \%$ $(\mathrm{w} / \mathrm{v})$ Triton X-100, $10 \mathrm{mM}$ EDTA, were applied to carbon film on copper grids. After a brief incubation, the specimen was blotted and washed with two droplets of $25 \mathrm{mM}$ Tris (pH 7.4), $20 \mathrm{mM} \mathrm{NaCl}, 2 \mathrm{mM}$ EDTA. It was negatively stained with $2 \%(\mathrm{w} / \mathrm{v})$ uranyl acetate, $0.1 \%$ $(\mathrm{w} / \mathrm{v})$ glucose. Areas with well embedded particles were photographed at a magnification of $59,000 \times$ with a Zeiss CEM 902 at $80 \mathrm{kV}$ using an electron dose of about $10 \mathrm{e}^{-} /$ $\AA^{2}$, discarding electrons scattered inelastically with $\Delta E>$ $\sim 15 \mathrm{eV}$. The objective lens defocus was chosen such that the first reversal of the phase contrast transfer function 
was at $\sim 0.05 \AA^{-1}$. The negatives were digitized with a Zeiss SCAI scanner at a step size of $21 \mu \mathrm{m}$, corresponding to $3.6 \AA$ at the level of the specimen.

The single particle image analysis was carried out in the context of the IMAGIC-5 software system, ${ }^{24}$ essentially as described by Schatz et al. ${ }^{25}$ and Serysheva et al. ${ }^{26}$ More than 10,000 single-molecular images were selected. They were subjected to the "alignment by classification" approach, by which bias towards any specific reference image is avoided. The resulting class averages were used as the starting set of reference images for iterative cycles of multi-reference alignment (MRA), multivariate statistical analysis (MSA) and classification. The MRA was performed using an "exhaustive alignment" procedure $^{27,28}$ based on resampling the images to polar coordinates. In general, classification resulted in classes with an average of ten to 25 members; five to $25 \%$ of the population was discarded.

After a stable set of classes was obtained, the relative projection angles (Euler angles $\beta$ and $\gamma$ ) were assigned to the class averages via angular reconstitution. ${ }^{29}$ For the first crude model, this assignment occurred via an ab initio bootstrap procedure leading to a decrease in the angle assignment errors. 3D reconstructions were calculated using the "exact-filter" back-projection algorithm. After reconstruction of the first crude $3 \mathrm{D}$ model, Euler-angle assignments were refined by means of an anchor set, obtained by reprojecting the model with angular increments of $30^{\circ}$, and a new $3 \mathrm{D}$ reconstruction was calculated. Different de novo analyses yielded essentially the same models.

One of the models thus obtained was used as input for an iterative procedure according to the following cycle: contouring of the model, reprojection with a particular angular increment to obtain references, MRA, MSA and classification, selection of class averages of well-defined appearance, Euler-angle assignment by means of an anchor set derived from the previous model, and 3D reconstruction. In early cycles of this iterative procedure, the anchor-set refinement was preceded by a semibootstrap procedure of Euler-angle assignment, using a subset of class averages with reliable Euler angles, well spread over the unit sphere, as a reference set. The correctness of the angles assigned was judged by assessing: (1) the fit between a class average and a mask derived from the previous model applying these angles and (2) the correlation between the input projection and the corresponding reprojection of the following model. The coverage of the unit sphere was also monitored. In the course of the iterative refinement procedure, the angular grid used to compute reprojections used as references for the MRA was gradually decreased from $20^{\circ}$ to $3^{\circ}$, and the angular increment used to establish the Euler angles was reduced from $5^{\circ}$ to $1^{\circ}$.

Initial image processing was performed without any symmetry imposed. During subsequent symmetry analyses, 2 or 4-fold symmetry was imposed at the stages of Euler-angle determination and 3D reconstruction. In each case, the refinement process was iterated to convergence or (in the case of 4-fold symmetry) until lack of convergence was demonstrated.

\section{Acknowledgements}

We thank Helga Clasen for expert technical assistance, Drs B. Sakmann, K. C. Holmes, and
R. R. Schröder for the use of MPI departmental facilities, and Doris Bader, Dr Günther Kümmerle, and Dr M. Schatz (IMAGE SCIENCE, Berlin) for helpful discussions. Financial support was provided by the Max Planck Society (D.R.M.), EU grant \#BIO4-CT96-0589 (D.R.M. \& K.K.), HHMI Biomedical Research Support Program to Medical Schools award \#76200-560801 (D.R.M.) and the Academy of Finland (K.K.).

\section{References}

1. Dingledine, R., Borges, K., Bowie, D. \& Traynelis, S. F. (1999). The glutamate receptor ion channels. Pharm. Rev. 51, 7-61.

2. Madden, D. R. (2002). The structure and function of glutamate receptor ion channels. Nature Rev. Neurosci. 3, 91-101.

3. O'Hara, P. J., Sheppard, P. O., Thogersen, H., Benezia, D., Haldeman, B. A., McGrane, B. et al. (1993). The ligand-binding domain of metabotropic glutamate receptors is related to bacterial periplasmic binding proteins. Neuron, 11, 41-52.

4. Armstrong, N. A. \& Gouaux, E. (2000). Mechanisms for activation and antagonism of an AMPA-sensitive glutamate receptor: crystal structures of the GluR2 ligand binding core. Neuron, 28, 165-181.

5. Kuner, T., Seeburg, P. H. \& Guy, H. R. (2003). A common architecture for $\mathrm{K}^{+}$channels and ionotropic glutamate receptors? Trends Neurosci. 26, 27-32.

6. Barry, M. F. \& Ziff, E. B. (2002). Receptor trafficking and the plasticity of excitatory synapses. Curr. Opin. Neurobiol. 12, 279-286.

7. Sun, Y., Olson, R., Horning, M., Armstrong, N., Mayer, M. \& Gouaux, E. (2002). Mechanism of glutamate receptor desensitization. Nature, 417, 245-253.

8. Horning, M. S. \& Mayer, M. L. (2004). Regulation of AMPA receptor gating by ligand binding core dimers. Neuron, 41, 379-388.

9. Ayalon, G. \& Stern-Bach, Y. (2001). Functional assembly of AMPA and kainate receptors is mediated by several discrete protein-protein interactions. Neuron, 13, 103-113.

10. Kuusinen, A., Abele, R., Madden, D. R. \& Keinänen, K. (1999). Oligomerization and ligand-binding properties of the ectodomain of the AMPA receptor subunit GluRD. J. Biol. Chem. 274, 28937-28943.

11. Pasternack, A., Coleman, S. K., Jouppila, A., Mottershead, D. G., Lindfors, M., Pasternack, M. \& Keinanen, K. (2002). $\alpha$-amino-3-hydroxy-5-methyl-4isoxazolepropionic acid (AMPA) receptor channels lacking the N-terminal domain. J. Biol. Chem. 277, 49662-49667.

12. Schorge, S. \& Colquhoun, D. (2003). Studies of NMDA receptor function and stoichiometry with truncated and tandem subunits. J. Neurosci. 23, 1151-1158.

13. Sobolevsky, A. I., Yelshansky, M. V. \& Wollmuth, L. P. (2004). The outer pore of the glutamate receptor channel has 2-fold rotational symmetry. Neuron, 41, 367-378.

14. Panchenko, V. A., Glasser, C. R. \& Mayer, M. L. (2001). Structural similarities between glutamate receptor channels and $\mathrm{K}^{+}$channels examined by scanning mutagenesis. J. Gen. Physiol. 117, 345-359.

15. Safferling, M., Tichelaar, W., Kümmerle, G., Jouppila, A., Kuusinen, A., Keinänen, K. \& Madden, D. R. 
(2001). First images of a glutamate receptor ion channel: oligomeric state and molecular dimensions of GluRB homomers. Biochemistry, 40, 13948-13953.

16. Miyazawa, A., Fujiyoshi, Y. \& Unwin, N. (2003). Structure and gating mechanism of the acetylcholine receptor pore. Nature, 423, 949-955.

17. Greger, I. H., Khatri, L. \& Ziff, E. B. (2002). RNA editing at Arg607 controls AMPA receptor exit from the endoplasmic reticulum. Neuron, 34, 759-772.

18. Greger, I. H., Khatri, L., Kong, X. \& Ziff, E. B. (2003). AMPA receptor tetramerization is mediated by $Q / R$ editing. Neuron, 40, 763-774.

19. Madden, D. R. (2002). The inner workings of AMPA receptors. Curr. Opin. Drug Discov. Dev. 5, 741-748.

20. Sobolevsky, A. I., Yelshansky, M. V. \& Wollmuth, L. P. (2003). Different gating mechanisms in glutamate receptor and $\mathrm{K}^{+}$channels. J. Neurosci. 23, 7559-7568.

21. Wenthold, R. J., Yokotani, N., Doi, K. \& Wada, K. (1992). Immunochemical characterization of the nonNMDA glutamate receptor using subunit-specific antibodies. Evidence for a hetero-oligomeric structure in rat brain. J. Biol. Chem. 267, 501-507.

22. Jespersen, L. K., Kuusinen, A., Orellana, A., Keinänen, K. \& Engberg, J. (2000). Use of proteoliposomes to generate phage antibodies against native AMPA receptor. Eur. J. Biochem. 267, 1382-1389.
23. Unwin, N., Miyazawa, A., Li, J. \& Fujiyoshi, Y. (2002). Activation of the nicotinic acetylcholine receptor involves a switch in conformation of the $\alpha$ subunits. J. Mol. Biol. 319, 1165-1176.

24. van Heel, M., Harauz, G. \& Orlova, E. V. (1996). A new generation of the IMAGIC image processing system. J. Struct. Biol. 116, 17-24.

25. Schatz, M., Orlova, E. V., Dube, P., Jäger, J. \& van Heel, M. (1995). Structure of Lubricus terrestris hemoglobin at $30 \AA$ resolution determined using angular reconstitution. J. Struct. Biol. 114, 28-40.

26. Serysheva, I. I., Orlova, E. V., Chiu, W., Sherman, M. B., Hamilton, S. L. \& van Heel, M. (1995). Electron cryomicroscopy and angular reconstitution used to visualize the skeletal muscle calcium release channel. Nature Struct. Biol. 2, 18-24.

27. Joyeux, L. \& Penczek, P. A. (2002). Efficiency of 2D alignment methods. Ultramicroscopy, 92, 33-46.

28. Sander, B., Golas, M. M. \& Stark, H. (2003). Corrimbased alignment for improved speed in singleparticle image processing. J. Struct. Biol. 143, 219-228.

29. van Heel, M. (1987). Angular reconstitution: a posteriori assignment of projection directions for $3 \mathrm{D}$ reconstruction. Ultramicroscopy, 21, 111-123.

Edited by W. Baumeister

(Received 28 April 2004; received in revised form 9 August 2004; accepted 20 September 2004) 